\title{
Severe Bleeding Tendency due to Excessive Fibrinolysis in Two Patients With Aortic Disease: Role of Tranexamic Acid in Non-Surgical Candidate
}

\author{
Pui Lun Yip ${ }^{a}$,, Sze Man June Lau ${ }^{a}$, Tsui Yin Jaime Lee
}

\begin{abstract}
Disseminated intravascular coagulation (DIC) is characterized by systemic activation of coagulation pathway which can lead to thrombosis and bleeding. Chronic DIC associated with aortic aneurysm or dissection commonly presents with bleeding symptoms with variable severity due to enhanced fibrinolysis. Patients with unexplained bleeding diathesis with DIC should include aortic disease as one of the differential diagnoses. This paper reports two patients with severe hemorrhagic complications due to aortic disease. Long-term prophylactic treatment is recommended if the underlying aortic disease cannot be surgically managed. Tranexamic acid is a potentially safe option to relieve bleeding tendency.
\end{abstract}

Keywords: Bleeding; Fibrinolysis; DIC; Aortic disease

\section{Introduction}

Disseminated intravascular coagulation (DIC) is characterized by systemic activation of coagulation system, resulting in consumption of clotting factors and platelets. Activation of fibrinolysis also occurs together with coagulation, but the degree of fibrinolysis depends on the underlying disease [1]. In patients with suppressed fibrinolytic DIC, widespread microthrombi are formed, leading to small vessels occlusion and organ dysfunction. In contrast, the enhanced fibrinolytictype DIC is associated with significant fibrinolysis resulting in bleeding diathesis. Uncommonly, chronic DIC secondary to aortic aneurysms or vascular malformation can be the underlying cause of enhanced fibrinolysis [2]. In patients with aortic dissection, the chronic coagulation activation due to exposure of collagen tissue and tissue factor in the false lumen locally leads to consumption of clotting factors and secondary

Manuscript submitted July 20, 2020, accepted July 28, 2020

Published online August 28, 2020

aDepartment of Medicine, Queen Elizabeth Hospital, Hong Kong, China ${ }^{\mathrm{b}}$ Corresponding Author: Pui Lun Yip, Department of Medicine, Queen Elizabeth Hospital, Hong Kong, China. Email: ypl741@ha.org.hk

doi: https://doi.org/10.14740/jmc3549 fibrinolysis. Subsequent depletion of platelets, clotting factors and fibrinogen may result in systemic bleeding tendency [3].

\section{Case Reports}

\section{Case 1}

A 78-year-old male patient had history of hypertension, diabetes mellitus, stage IV chronic kidney disease and infra-renal abdominal aortic aneurysm (AAA) with endovascular stent. Type II Endo-leak was noted at the level of bifurcation on follow-up computed tomography (CT) scan after stenting. He experienced easy bruising after the operation but did not seek medical attention until 4 years later, when he presented to orthopedics with right knee non-traumatic hemarthrosis after hiking. Initial blood tests revealed mild thrombocytopenia (platelets $99 \times 10^{9} / \mathrm{L}$ ), prothrombin time (PT) $13.8 \mathrm{~s}$ (reference range (RR): 10.7 - 13.1 s) and activated partial thromboplastin time (APTT) $27.1 \mathrm{~s}$ (RR: 25.8 - $33.8 \mathrm{~s}$ ). The hemarthrosis resolved gradually, but his spontaneous bruises persisted. Investigations for bleeding tendency including factor V, VII, VIII, IX, X, XI, XIII, Von Willebrand antigen and ristocetin cofactor activity were performed and all were within normal limits. Repeated D-dimer was elevated to $>5,000 \mathrm{ng} / \mathrm{mL}$, and fibrinogen was reduced to $1.36-1.4 \mathrm{~g} / \mathrm{L}$ (RR: $1.71-3.38 \mathrm{~g} / \mathrm{L}$ ). He had a second episode of spontaneous right knee hemarthrosis 6 months later. Hemarthrosis subsided with regular use of tranexamic acid. Fibrinogen level rose to $3.16 \mathrm{~g} / \mathrm{L}$ after a few days of regular tranexamic acid and it was then stopped. Three months following the second episode of hemarthrosis, he presented to the emergency department with sudden onset of unconsciousness without any trauma, and his blood pressure was well-controlled. Urgent CT scan of head showed extensive intraventricular hemorrhage. The patient passed away 2 days after admission.

\section{Case 2}

A 63-year-old woman had no medical problems apart from being a hepatitis B carrier. She was admitted for sudden onset of severe chest pain. CT revealed type A aortic dissection extending distally from the aortic root to the left common iliac artery. She developed pulseless electrical activity just prior to skin 


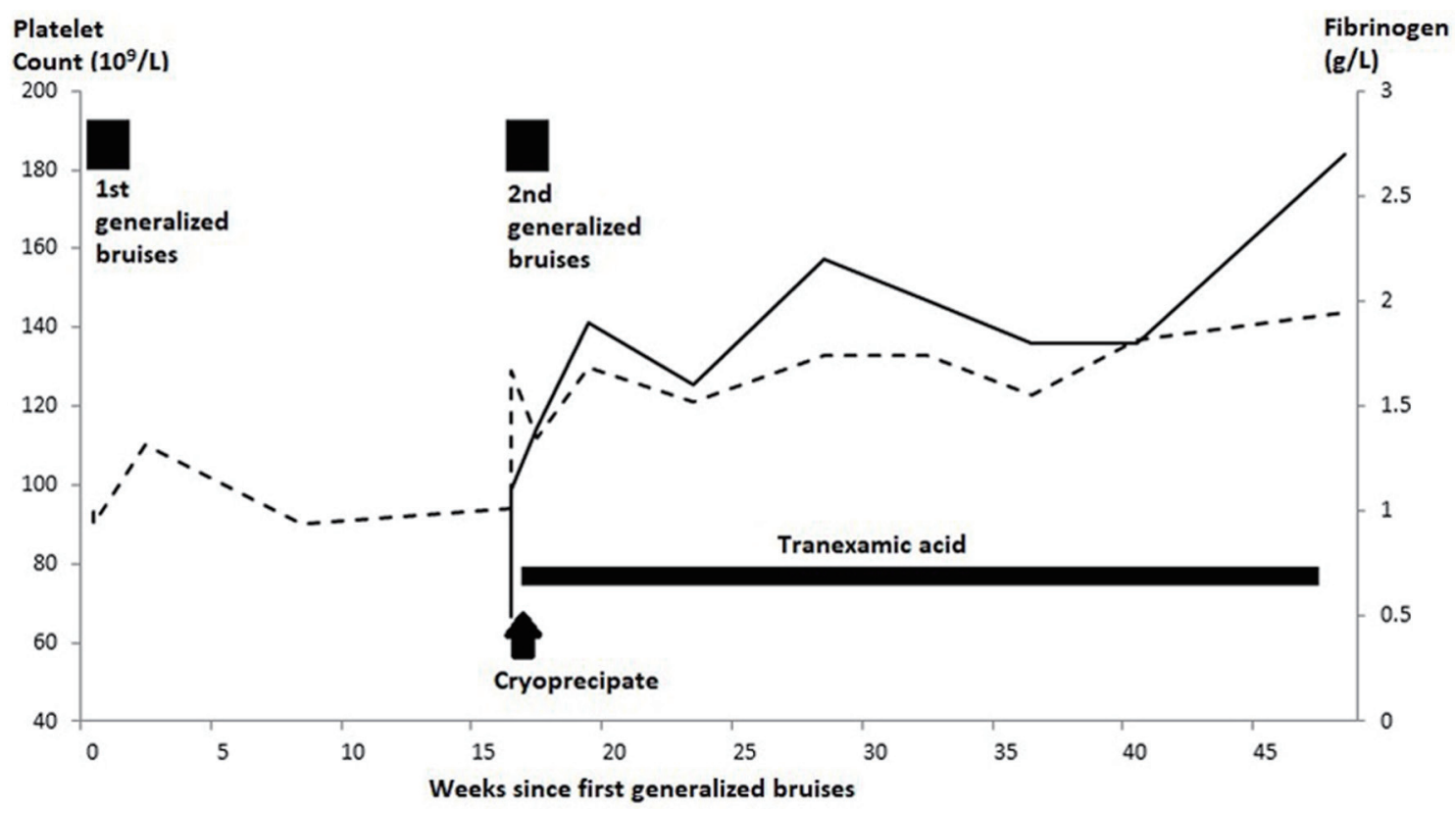

Figure 1. Clinical course of treatment and improvement of DIC-related parameters in patient 2. DIC: disseminated intravascular coagulation. Dotted line denote platelet count, solid line denote fibrinogen.

incision due to cardiac tamponade. Emergency resection and replacement of ascending thoracic aorta was performed. She managed to have a good recovery without neurological sequelae. She presented 6 months after discharge with generalized spontaneous bruises. Her hemoglobin dropped from 11.1 to $8.4 \mathrm{~g} / \mathrm{dL}$ and her platelets count was $90 \times 10^{9} / \mathrm{L}$. Clotting profile was normal except for mild prolongation of APTT $14.5 \mathrm{~s}$ and elevated D-dimer $>5,000 \mathrm{ng} / \mathrm{mL}$. She experienced hematuria and managed conservatively. Four months after the first episode of bruises, she suffered from extensive bruises over her limbs. Laboratory data indicated anemia, thrombocytopenia (hemoglobin $5.7 \mathrm{~g} / \mathrm{dL}$, thrombocytopenia $90 \times 10^{9} / \mathrm{L}$ ) and abnormal clotting profile (APTT $38.5 \mathrm{~s}$, PT $16.5 \mathrm{~s}$, fibrinogen $0.5 \mathrm{~g} / \mathrm{L}$ and D-dimer $>5,000 \mathrm{ng} / \mathrm{mL}$ ) (Fig. 1). Clotting factors and von Willebrand assays as performed in case 1 were all normal. The International Society on Thrombosis and Hemostasis (ISTH) DIC score was 6, consistent with overt DIC. In view of the development of bleeding tendency after aortic dissection, thrombocytopenia, chronically elevated D-dimer and low fibrinogen, DIC with excessive fibrinolysis secondary to aortic dissection was suggested. Bleeding tendency was stopped by cryoprecipitate to replenish fibrinogen together with regular tranexamic acid. Follow-up CT scan showed aortic dissection of similar extent - both true and false lumens were patent, with slower perfusion over false lumen. The option of surgical repair was discussed with the surgeons to ameliorate the chronic DIC and bleeding tendency, but the surgical risk was deemed to be too high for the patient. Therefore, long term tranexamic acid (750 mg daily) was prescribed to control the fibrinolysis. Eight months after taking regular tranexamic acid, the previously reduced level of fibrinogen recovered to $2.7 \mathrm{~g} / \mathrm{L}$ and the platelets count improved to $144 \times 10^{9} / \mathrm{L}$. She did not experience any recurrence of bruises or bleeding symptoms.

\section{Discussion}

Fibrinolysis is activated in parallel with coagulation to regulate and limit clot formation. In some clinical conditions (e.g., severe trauma, post-partum hemorrhage, uncontrolled acute promyelocytic leukemia, etc.), excessive fibrinolysis predominates and results in bleeding tendency. A study involving 76 pre-operative patients with aortic aneurysms showed elevation of fibrin-degradation products in $39 \%$ of the patients, while clinically overt DIC occurred in up to $4 \%$ [4]. Nevertheless, it could be difficult for clinicians to establish the association of DIC, bleeding and vascular disease if they were unfamiliar with the condition, and particularly if patients had remote history of aortic surgery. Both cases 1 and 2 showed bleeding complications and laboratory findings of markedly elevated level of D-dimer, thrombocytopenia and hypofibrinogenemia. DIC-related bleeding should be considered as a potential cause of unusual bleeding in patients with aortic disease or after aortic surgery. Apart from aortic dissection and stenting with endoleak, there were no other identified causes that triggered coagulopathy in our patients. There is no single gold standard laboratory diagnostic test for DIC with enhanced fibrinolysis. Investigations usually show elevated levels of D-dimer (or fibrin and fibrinogen degradation product), plasmin-alpha 2-plasmin inhibitor complex and thrombin-antithrombin complex coupled with hypofibrinogenemia. Fibrinogen level and clinical symptoms responded to treatment quickly as shown in case 2, hence fibrinogen could be used as a marker for monitoring the activity of DIC-fibrinolysis.

Treatment for DIC should be focused on treating the underlying condition [5]. Surgical intervention (e.g., replacement surgery or repair) is the primary treatment for chronic DIC caused by AAA or dissection. In patients presenting with acute bleeding complication, supportive measures to replace the defi- 
cient clotting factors and platelets is the mainstay of treatment. Both of our cases have the bleeding tendency drastically ameliorated by normalizing the fibrinogen level. Antifibrinolytic agents such as tranexamic acid, is generally not recommended for patient with DIC due to the risk of thromboembolism. However, it could be a reasonable option for severe bleeding patients with DIC and hyperfibrinolysis [5]. When surgical or endovascular intervention for the aortic disease is not possible, long term medical treatment would be needed to control the chronic DIC and fibrinolysis. There is no standard systemic therapy for aortic enhanced fibrinolysis-DIC; anticoagulation [6], antifibrinolytics [7], or a combination of both [8] have been reported to be successful in halting the consumption of coagulation factors. The main purpose of anticoagulation is to inhibit thrombus generation, thereby the cycle of thrombus formation and destruction would be interrupted. On the other hand, tranexamic acid inhibits the enzymatic breakdown of fibrin by plasmin, thus reducing the activity of fibrinolysis. In case 2, coagulopathy as measured by fibrinogen level was quickly reversed by tranexamic acid and she remained free from bleeding complications for more than 8 months. We believed that the long-term use of tranexamic acid is reasonable in view of her recurrent spontaneous bleeding diathesis. This approach of using long term tranexamic acid has been reported to successfully control DIC with excessive fibrinolysis in a patient with chronic renal failure [9]. As shown in case 1, who suffered from spontaneous intracranial hemorrhage after discontinuation of tranexamic acid, bleeding complications can recur if medical treatment is not maintained. Long-term treatment is therefore essential to ensure that bleeding tendency is controlled if the underlying aortic disease cannot be surgically managed.

\section{Conclusions}

In conclusion, DIC with enhanced fibrinolysis should be considered as the underlying cause of abnormal bleeding in patients with aortic disease. The risk of bleeding persists in patients without surgical correction, therefore long-term medical treatment is warranted. Tranexamic acid can be used in acute bleeding episodes to stop bleeding, and it can also be a feasible option for maintenance therapy.

\section{Acknowledgments}

None to declare.

\section{Financial Disclosure}

No funding was received. None of the authors have disclosures relevant to this manuscript.

\section{Conflict of Interest}

None to declare.

\section{Informed Consent}

The manuscript has been sufficiently de-identified to protect the patient. Patient 1 has since died and is unable to provide informed consent. Written informed consent was obtained from the patient 2 for publication of this case report.

\section{Author Contributions}

Pui Lun Yip, Sze Man June Lau and Tsui Yin Jaime Lee treated the patients, wrote and approved the manuscript.

\section{Data Availability}

The authors declare that data supporting the findings of this study are available within the article.

\section{References}

1. Asakura H. Classifying types of disseminated intravascular coagulation: clinical and animal models. J Intensive Care. 2014;2(1):20.

2. Levi M, Scully M. How I treat disseminated intravascular coagulation. Blood. 2018;131(8):845-854.

3. Thompson RW, Adams DH, Cohen JR, Mannick JA, Whittemore AD. Disseminated intravascular coagulation caused by abdominal aortic aneurysm. J Vasc Surg. 1986;4(2):184-186.

4. Fisher DF, Jr., Yawn DH, Crawford ES. Preoperative disseminated intravascular coagulation associated with aortic aneurysms. A prospective study of 76 cases. Arch Surg. 1983;118(11):1252-1255.

5. Levi M, Toh CH, Thachil J, Watson HG. Guidelines for the diagnosis and management of disseminated intravascular coagulation. British Committee for Standards in Haematology. Br J Haematol. 2009;145(1):24-33.

6. Kawano H, Hata T, Uda A, Maemura K. Use of rivaroxaban for the effective management of disseminated intravascular coagulation associated with abdominal aortic aneurysm. Intern Med. 2015;54(20):2625-2628.

7. Uzuka T, Nakamura M, Kuroda Y, Watanabe N. The use of an anti-fibrinolytic agent to reduce endoleak following endovascular repair of multiple aortic aneurysms. Interact Cardiovasc Thorac Surg. 2015;21(2):266-268.

8. Ontachi Y, Asakura H, Arahata M, Kadohira Y, Maekawa M, Hayashi T, Yamazaki M, et al. Effect of combined therapy of danaparoid sodium and tranexamic acid on chronic disseminated intravascular coagulation associated with abdominal aortic aneurysm. Circ J. 2005;69(9):11501153.

9. Kimura SI, Odawara J, Aoki T, Yamakura M, Takeuchi M, Watanabe Y, Matsue K. Use of tranexamic acid for disseminated intravascular coagulation with excessive fibrinolysis associated with aortic dissection in a patient with chronic renal failure. Int J Hematol. 2009;89(4):549-552. 